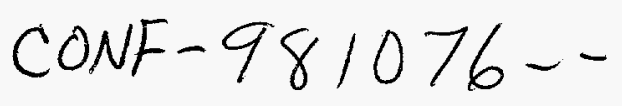

\title{
Recent Highlights of X-ray Magnetic Scattering Studies from Surfaces
}

\author{
G.M. WATSON ${ }^{1}$, Doon GIBBS ${ }^{2}$, and G.H. LANDER ${ }^{3}$ \\ 1 Department of Physics, University of Marylana, Baltimore Caunty. 1000 Hilltop Circle, Baltimore, MD 21250 \\ 2 Department of Physics, Brookhaven National Laboratory, Upton, New York 11973-5000 \\ 3 European Institute of Transuramic Elements, Postfach 2340, D-76125 Karlsruhe, Germany
}

In this work, recent studies of surface magnetism, as observed by $\mathrm{x}$-ray scattering techniques, are described. The experiments were concerned with uranium dioxide crystals for which $\mathrm{x}$-ray resonance effects enharice the small magnetic signal from the surface. The main result is that, in contrast to the bulk which exhibits a discontinuous magnetic ordering transition, both the (001) and (111) surface layers order continuously. This is reminiscent of the general phenomenon of surface wetting, but had not been previously observed for magnetic materials. Magnetic reflectivity studies show further that the near-surface magnetic layers are more disordered than layers deep in the bulk, even at low temperatures.

Keywords: surface magnetism, X-ray scattering

\section{Introduction}

During the last 10 years, $\mathrm{x}$-ray magnetic scattering techniques using synchrotron radiation have blossomed, especially in studies of rare earth metals and actinides, including bulk materials, thin films and compounds. These studies have especially benefited from the use of the resonance and polarization properties of the cross section when the incidert $\mathrm{x}$-ray energy is tuned near as $\mathrm{L}$ or $\mathbf{M}$ absorption edge. Brief reviews of these techniques and recent applications may be found in references 1 and 2 . Non-resonant magnetic scattering has also continued to develop, most notably in studies of transition element magnetism using incident photons of $240 \mathrm{keV}^{3)}$ and in studies of the separation of the orbital and spin magnetization densities. ${ }^{4}$ In the former case the enhancement to the signal comes from the increased penetration (up to cms) possible with high energy photons. Although the strengths of x-ray magnetic scattering techniques sometimes overlap those of neutron diffraction, they are generally complementary, and include high $Q$ resolution, sensitivity to lattice modulations, small beam size and useful polarization and resonance properties.

For these reasons, $x$-ray magnetic scattering studies of the magnetic structure of rare earth and actinide materials (including thin films) have almost become routine..$^{5-9)} \mathrm{A}$ recent review of $x$-ray scattering studies of rare-earth metals has been given by McMorrow, Gibbs and Bohr (1998). ${ }^{10)}$ New kinds of experiments have been concerned with critical properties, characterized near magnetic ordering transformations ${ }^{11,12)}$ and with the use of circularly polarized incident beams. ${ }^{\text {(3) }}$ In addition, these techniques have been extended to surfaces and interfaces. ${ }^{14.15)}$ Very recently, resonant scattering techniques have been applied to the direct observation of orbital ordering in transition metal oxides. ${ }^{15)}$

In the following, we briefly describe recent efforts to probe long-ranged magnetic order at surfaces by $x$-ray and neutron diffraction techniques, ${ }^{17-26)}$ following many earlier studies by low energy electron diffraction. ${ }^{27}$ ) The main motivation has been to discover how bulk magnetic structures are modified near a surface, where the crystal symmetry is broken. Some basic questions of interest include: Does the magnetization profile at the surface follow that of the electronic charge density? Does the magnetic critical behavior near a surface differ from that in bulk? What is the relationship between chemical and magnetic interfacial roughness?

Neutron diffraction has been the traditional probe of magnetic structure, and was first applied to surfaces by Felcher, et al. ${ }^{12)}$ to obtain magnetic depth profiles of various materials using reflectivity techniques. Subsequently, AlUsta, et al. ${ }^{24)}$ observed weak magnetic signals at glancing incidence in $\mathrm{MnF}_{2}$. Enormous, related progress has been made in studies of interfacial magnetism through the use of multilayers (see e.g., ref. 12) although the interfacial strain and bilayer coupling introduce additional complexities.

In this work, $\mathrm{x}$-ray scattering studies of magnetic ordering have been carried out on the (001) and (111) surfaces of $\mathrm{UO}_{2}{ }^{17-20)}$ The initial aim was simply to observe a pure magnetic truncation rod (defined below) and, if possible, to then explore the near-surface magnetism directly. The motivation for choosing $\mathrm{UO}_{2}$ was two-fold: first, to take advantage of the large resonant enhancements of the magnetic cross-section which occur when the incident photon energy is tuned near the uranium $\mathrm{M}_{4}$ absorption edge. ${ }^{293)}$ This work follows earlier observations of the magnetic reflectivity in the soft $x$-ray range by Kao, et al. ${ }^{26}$ ) 
and complements magnetic circular dichroism studies, which are centered at zero momentum transfer. ${ }^{30,31)}$

By way of background, $\mathrm{UO}_{2}$ has the face-centered cubic fluorite lattice and a triple- $Q$ antiferromagnetic structure $\left(T_{n}=30.2 K\right)$. The surface diffraction pattern consists of a set of rods of scattering (called truncation rods) which pass through all of the allowed bulk Bragg points, and lie parallel to the surface normal direction. ${ }^{32)}$ The variation of the $x$-ray intensities along the chemical truncation rods is determined by the decay of the electronic charge density near the surface, and may be used to model the surface structure. Magnetic truncation rods may be defined similarly, with the intensity variation now determined by the magnetic structure near the surface. ${ }^{26)}$

Scans of the $(01 \mathrm{~L})$ magnetic truncation rod taken along the surface normal direction of a $\mathrm{UO}_{2}(001)$ crystal are shown in Fig. 1 for temperatures increasing from 10 to 30.2 $\mathrm{K}^{17-19)}$

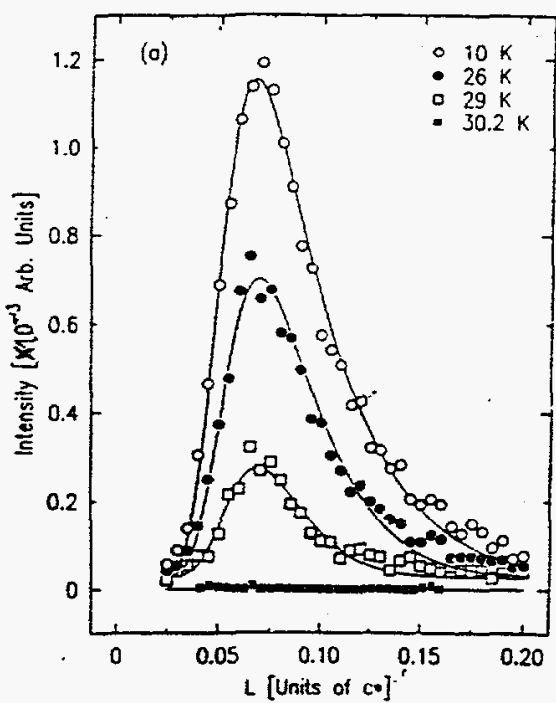

Fig. 1. Temperature dependence of the (01L) magnetic truncation rods of $\mathrm{UO}_{2}(001)$.

The L-dependence of the lineshape is characteristic of a truncation rod of the chemical lattice with the peak at $\mathrm{L}=0.07$ corresponding to the critical angle. Separate experiments monitoring the energy and polarization dependence of these profiles have shown that they arise from pure magnetic scattering. This is also consistent with the temperature dependence of the scattering which decreases with increasing temperature, untii it disappears at $\mathrm{T}_{\mathrm{N}}=30.2 \mathrm{~K}$. Signal rates as high as $1000 / \mathrm{sec}$ have been observed at the critical angle using undulator and wiggler beamlines. More exciting, recent improvements in actinide surface preparation techniques ${ }^{19}$ ) suggest that increases by as much as a factor fifteen are readily available!
Fig. 2 shows the temperature dependence of the magnetic scattering intensity obtained at two positions along the (01L) magnetic truncation rod, and at the bulk (001) reflection. $^{17-19)}$ The discontinuity observed at $\mathrm{T}=30.2 \mathrm{~K}$ for

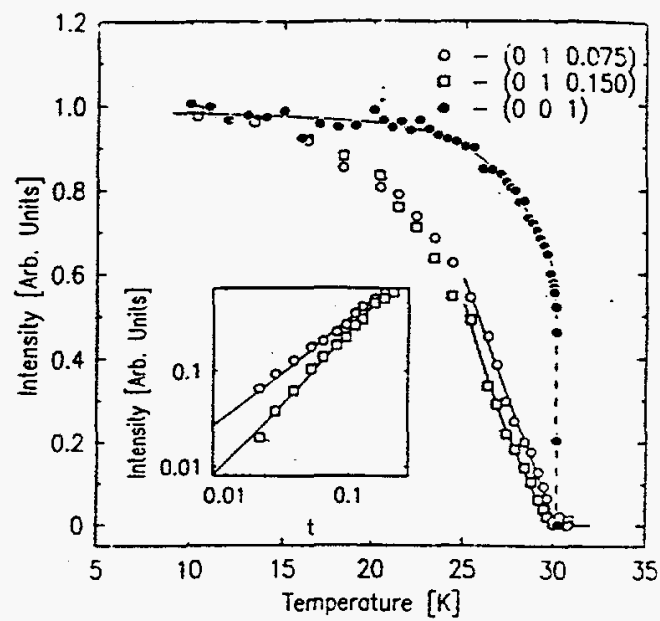

Fig. 2. Temperature dependence of the magnetic scattering of bulk (001) reflection (closed symbols) and of the (01L) truncation rod at two values of $\mathrm{L}$. Inset: $\mathrm{Log}-\log$ pilot of the rod intensity vs. reduced temperature.

the scattering at the $(001)$ reflection is characteristic of bulk behavior. In contrast, the magnetic scattering intensities obtained along the truncation rods fall more slowly to zero as $T_{N}$ is approached from below-indeed, they appear continuous. This behavior is reminiscent of the general phenomenon of surface-induced disorder, wherein a partially disordered layer of crystalline phase wets the nearsurface volume below $T_{c}$ and grows logarithmically in thickness as $T$ approaches $T_{c^{-}}{ }^{33)}$ Similar results have been observed earlier in X-ray structural studies of the orderdisorder transition in $\mathrm{Cu}_{3} \mathrm{Au},{ }^{34)}$ however, this is the first such observation for a magnetic material. Within Landau theory, these theories predict that the temperature dependence of the order parameter should follow a power law in reduced temperature under specific conditions. ${ }^{33}$ ) Fits of the magnetic scattering intensity to a power-law form are shown for two values of $\mathrm{L}$ by the solid lines in Fig. 2 . The fits are satisfactory, and yield exponents increasing between (1/2) and 1, depending on $L$. This behavior is qualitatively consistent with surface induced disorder provided the diffuse scattering at the interface is described within the Gaussian approximation-which is a satisfactory approximation for these data.

Measurements of the magnetic specular reflectivity allow further insight into the interfacial magnetic structure. This is illustrated in Fig. 3, which shows the pure magnetic reflectivity obtained from a $\mathrm{UO}_{2}(001)$ surface in the 


\section{DISCLAIMER}

This report was prepared as an account of work sponsored by an agency of the United States Government. Neither the United States Government aor any agency thereof, nor any of their employees, makes any warranty, express or implied, or assumes any legal liability or responsibility for the accuracy, completeness, or usefulness of any information, apparatus, product, or process disclosed, or represents that its use would not infringe privately owned rights. Refereace berein to any speciric commercial product, process, or service by trade name, trademark, manufacturer, or otherwise does not necessarily constinute or imply its endorsement, recommendation, or favoring by the United States Governmeat or any agency thereof. The views and opinions of authors expressed herein do not pecessarily state or reflect those of the. United States Government or any agency thereof. 


\section{DISCLAIMER}

Portions of this document may be illegible in electronic image products. Images are produced from the best available original document. 
neighborhood of the bulk (001) reflection at two different tcmperatures. ${ }^{17)}$ The intensity of the magnetic scattering at the bulk reflection is over $300,000 / \mathrm{sec}$ and falls off by five orders of magnitude within a few tenths of an inverse Angstrom. The wings of the scattering are clearly much weaker at higher temperatures near $T_{N}=30.2 \mathrm{~K}$ (closed circles) than at $10 \mathrm{~K}$ (open circles). This suggests that the magnetic interface is more diffuse ac higher temperatures,

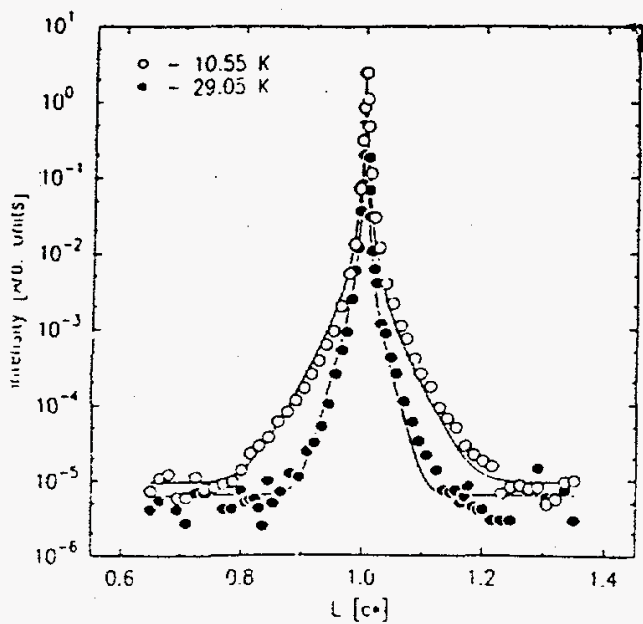

Fig. 3. Specular magnetic reflectivity profiles in the neighborhood of the bulk (001) reflection.

since, broadly speaking, a sharper reflectivity profile in reciprocal space is consistent with a more extended density profile in real space.

Fits of the specular and truncation rod intensities ${ }^{17)}$ were made to a model of the magnetic interface, consisting of an interfacial width $\chi$ and thickness $L$ of magnetically disordered volume (see Fig. 4). The magnetic profiles were otherwise assumed to match the profile of the electronic charge density, which was also determined using refiectivity techniques. The latter correspond closely to ideal termination, supplemented by a thin absorbing layer. The best fits are shown by the solid lines in Figs. 3 and 4. The thickness of the disordered layer $L$ was found to be relatively insensitive to variations in the temperature, and could be fixed at a value of about $13 \AA$. The corresponding value of the interfacial width at low temperatures $(10 \mathrm{~K})$ was about $6 \AA$. Thus, in contrast to the electronic charge density, the magnetic structure is disordered very near the surface, and has a more rounded profile, even at $10 \mathrm{~K}$ in the magnetically ordered phase. For increasing temperatures, the interfacial width exhibited a dramatic increase, starting at about $25 \mathrm{~K}$ and diverging as $\mathrm{T}$ approached $T_{\mathrm{N}}$. This is illustrated in Fig. 5, where the fitted widths are plotted versus temperature for two different $\mathrm{UO}_{2}$ (001) samples. $\left.{ }^{17}\right)$ This behavior is again qualitatively consistent with the

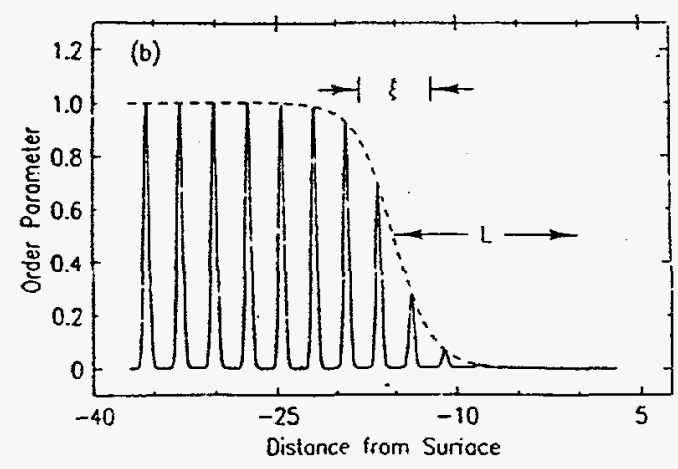

Fig. 4. Model of the magnetic interface fitted to the reflectivity profiles. The chemical surface is located at 0 on the $x$-axis.

theory of surface induced disorder, which predicts a logarithmic divergence of the widths.

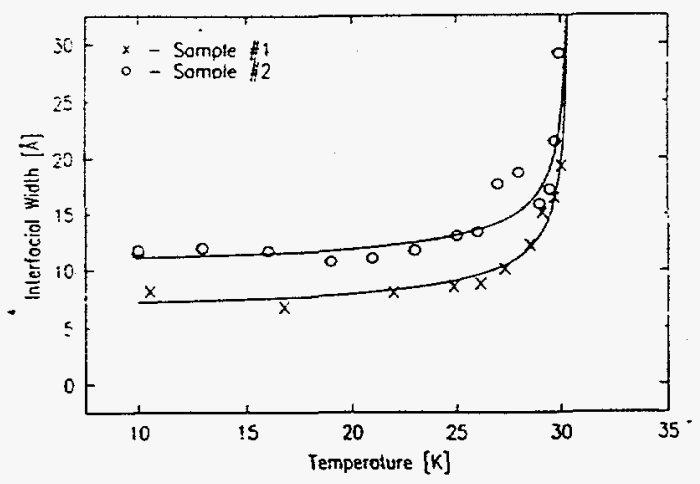

Fig. 5. Temperature dependence of the interfacial magnetic widths extracted from reflectivity experiments of two different samples.

It is important to add that recent studies of $\mathrm{UO}_{2}(111)$ surfaces have produced strikingly similar results to those noted here for the (001) surface, ${ }^{19)}$ including : a continuous magnetic ordering transition and increased near-surface disorder. Especially encouraging, the signal rates are large enough that it appears possible to characterize the transverse lineshapes of the reflectivity at temperatures near $T_{N}$. Preliminary results suggest the possibility of magnetic roughening transition as $T$ approaches $T_{\mathrm{N}}$. It is also worth noting in this regard that the assembled results for $\mathrm{UO}_{2}$ surfaces stand in intriguing contrast to the results of magnetic reflectivity studies of Uas and UP surfaces ${ }^{21)}$ in which $2 \mathrm{D}$ magnetic layer was found to persist near the surface at and just above $T_{N}$. Of course the bulk magnetic structure of $\mathrm{UO}_{2}$ differs from that of these materials, but it 
remains to understand the detailed origin of the surface behavior.

In summary, the first x-ray experiments carried out on $\mathrm{UO}_{2}(001)$ and (111) magnetic surface layers already reveal a number of surprising and interesting results, including a continuous magnetic ordering transition and increased nearsurface magnetic disorder. This occurs even though the chemical interfaces are nearly ideally terminated. Because these observations have been repeated on two different surface orientations and on several samples, these properties may be more general than was previously anticipated. Answering that question is one focus of continuing experiments. Finally, the present data and discussion illustrate the range of possibilities open to the characterization of magnetic interfaces using these techniques, and have played a seminal role in stimulating the field. There is already a diversity of experiments underway on these and related issues in actinide, ${ }^{28,2 l)}$ rare earth $^{22)}$ and transition element ${ }^{22,23)}$ surface magnetism at $\mathrm{x}$ ray beamlines around the world.

\section{Acknowledgements}

Work performed at Brookhaven is supported by the U.S. Department of Energy under contract No. DE-AC02$98 \mathrm{CH} 10886$.

\section{References}

1) D. Gibbs: Synchrotron Radiation News 5 (1992) 18.

2) W. Stirling, and G. H. Lander,: Synchrotron Radiation News 5 (1992) 17.

3) J. Schneider: Synchrotron Radiation News 8(2) (1995) 26.

4) V. Fernandez, et al.: Phys. Rev. B57 (1998) 7870.

5) J.P. Hill, B. Sternlieb, D. Gibbs, C. Detlefs, A. Goldman, C. Stassis, P. Canfield, B. Cho: Phys. Rev. B53 (1996) 3487.

6) C. Detlefs, et al.: Phys. Rev. B53 (1996) 6355.

7) G. Helgesen, J.P. Hill, T.R. Thurston, D. Gibbs, J. Kwo, M. Hong: Phys. Rev. B50 (1994) 2990.

8) G. Helgesen, et al.: Phys. Rev. B52 (1996) 9446.

9) G. Helgesen, et al.: Phys. Rev. B56 (1997) 2635.

10) McMorrow, D. Gibbs, Bohr: Handbook of Rare Earth Metals (in press).

11) W. J. Nuttall, S. Langridge, W.G. Stirling, G.H. Lander, B. Lebeck, and O. Vogt: Phys. Rev. B (1996).

12) T. Thurston, G. Helgesen, J.P. Hill, D. Gibbs, B.D. Gaulin, P. Simpson: Phys. Rev. B49 (1994) 15730.

13) C. Sutter, G. Grübel, C. Vettier, F. de Bergerin, A. Stunault, D. Gibbs, C. Giles: "to be published in Phys. Rev. B."
14) G. M. Watson, et al.: Physics (Amsterdam) 221B (1996) 405.

15) S. Ferrar, et al.: Phys. Rev. Lett. 77 (1996) 747.

16) Murakami, et al.: Phys. Rev. Lett. 80 (1998) 1932; op cit 81 (1998) 582.

17) G. M. Watson, D. Gibbs, G.H. Lander, B. D. Gaulin, L.E. Berman, Hj. Matzke, W. Ellis: Phys. Rev. Lett. 77 (1996) 751; G.M. Watson, et al., Phys. Rev. B (submitted).

18) G. P. Felcher, et al.: Phys Rev. Lett. 52 (1984) 1539; Physica B192 (1993) 137.

19) G. M. Watson, D. Gibbs, G.H. Lander, Hj. Matzke, C. Vettier: unpublished

20) Search and Discovery, Physics Today, November 1996) 21.

21) A. Stunault, S. Langridge, C. Vettier, D. Gibbs, N. Bernhoeft: Phys. Rev. B55 (1997) 423.

22) S. Ferrer, et al.: Phys. Rev. Lett. 77 (1996) 747; ibid, Phys. Rev. B56 (1997) 9848.

23) J. F. Mackay, et al.: Phys. Rev. Lett. 77 (1996) 3925.

24) K. Al-Usta, et al.: Physics (Amsterdam) 173B (1991) 65.

25) C.C. Kao, et al.: Phys. Rev. Lett. 65 (1990) 373.

26) A. Fasolino, et al. Phys. Rev. B47 (1993) 3877.

27) P.W. Palmberg, et al. Phys. Rev. Lett. 21 (1968) 682; R.E. Dewames and T. Wolfran, ibid 22 (1969) 137; R.J. Celotta, et al.: ibid 43 (1979) 728; S.F. Alvarado, et al. ibid 48 (1982) 51; B. Dauth, et al. Surf. Sci. 189/190 (1987) 729.

28) C.P. Flynn and M. Salamon: Handbook on Physics and Chemistry of Rare Earths, Gschneidner and Eyring (Eds.), Vol. 22, pp. 1 (Elsevier).

29) D. B. McWhan, et al.: Phys. Rev. B42 (1990) 6007

30) J. G. Tobin, et al.: Phys. Rev. Lett. 68 (1992) 3642.

3i) J. Stohr, et al.: Science 259 (1992) 658.

32) I. K. Robinson: Phys. Rev. B33 (1981) 3830.

33) R. Lipowsky and W. Speth: Phys. Rev. B28 (1983) 3983.

34) H. Dosch, et al.: Phys. Rev. Lett. 60 (1988) 2382. 